\title{
Exploiting Trauma as an Artistic Medium
}

\author{
Sean Rogg \\ Founder and Artistic Director \\ The Waldorf Project \\ London \\ UK \\ http://www.seanrogg.com \\ seanrogg@hotmail.com
}

\section{OVERVIEW}

The Waldorf Project (www.waldorfproject.com) is a genre defying and radical immersive art performance that uses combined technologies and emotional manipulation to develop new ways of implementing algorithmic methods which manipulate a group in order to explore a creative vision.

Created and directed by artist Sean Rogg, the Project unites the most pioneering practitioners in the worlds of environment design, product design, spatial design, sound design, choreography, textile design, and gastronomy, to engender the birth of a new art form: a rare synergy, a singular collected sensory reaction, or 'sixth sense' of emotion.

Developed over 5 years, though a series of 'Chapters' Sean has created a new artistic language, the complete fusion of the senses, binding all perceptions together into one. His future facing vision is one that presents a radical departure from the current trend of digital immersive art and has been highlighted by global trend forecasters as one of the most 'forward thinking art experiences of our time'.

Conceptually and physically pioneering new ways to perceive spatial design, The Waldorf Project marries cutting-edge design with radical architecture that responds and changes its emotional state, depending on the behavior of its inhabitants. The boundaries of how and when are contextualized within this intangible environment. It heralds a new level of manipulation; as strangers to a 'brave new world', participants are prompted to learn anew, and to react (and act) in ways unfamiliar. Lost in the vacuum, they are guided to move both as individuals and as a newly formed cumulative organism.

As the next 'chapter' BARZAKH: approaches, founder and creative director Sean Rogg talks us through the journey and development of his methods. One in which he has learnt to use controlled trauma to cleanse participants and reframe their entire reality.

"We are not thinking beings that feel; we are feeling beings that think."

- Antonio Domasio, Professor of Neuroscience

Chapter Four/BARZAKH will take place in November 2017.

\section{BIOGRAPHY}

Sean Rogg is Founder and Artistic Director of the The Waldorf Project. He is a British artist working in the field of video, photography and performance. His work has been exhibited at The Wapping Project, Bloomberg SPACE and ICA in London, as well as part of TinaB Biennale in Prague, at Modern Art Oxford, Kunsthal Charlottenborg in Copenhagen, The CCA in Tel Aviv, Galerie Mathias Güntner in Hamburg and Galleri Kleerup in Stockholm. 


\section{IMAGES}

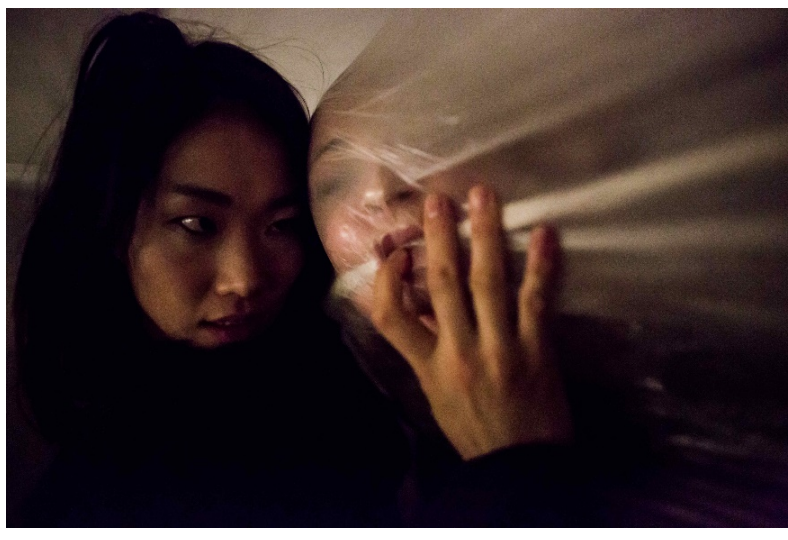

Figure 1: A guest at stage 4 (rebirth) gagging/suffocation and tear through plastic membrane

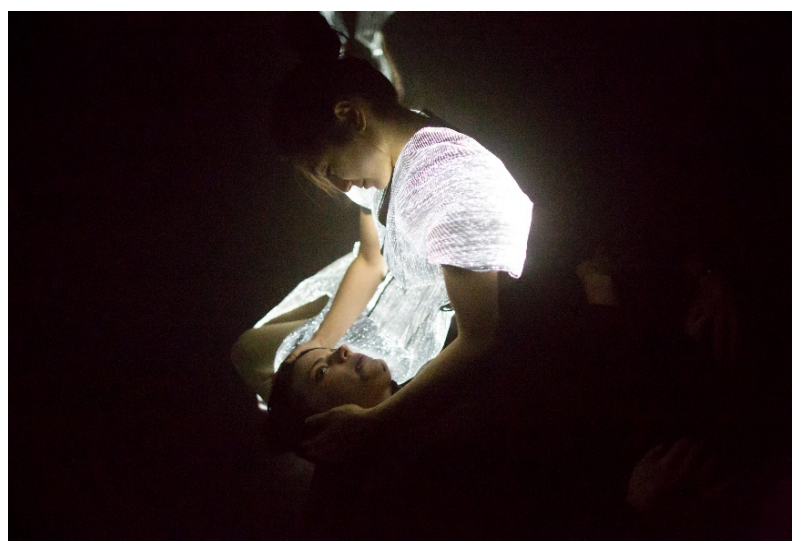

Figure 2: All 40 guests in a meditative state awaiting to be "connected"

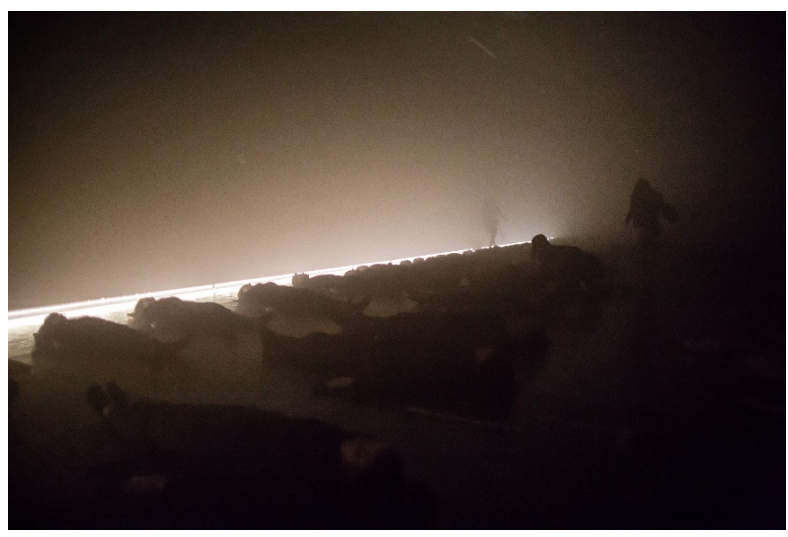

Figure 3: A guest in the final sequence being fed "Energy"

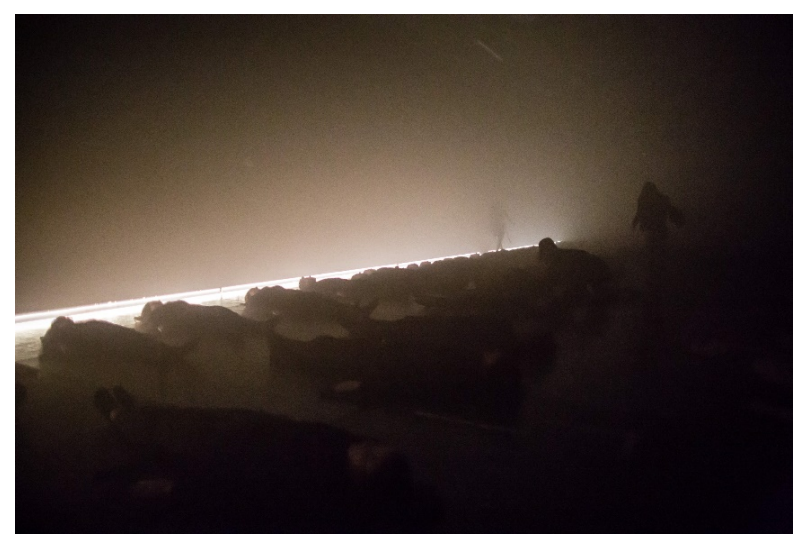

Figure 4: All 40 guests in the second stage in a group/isolation state

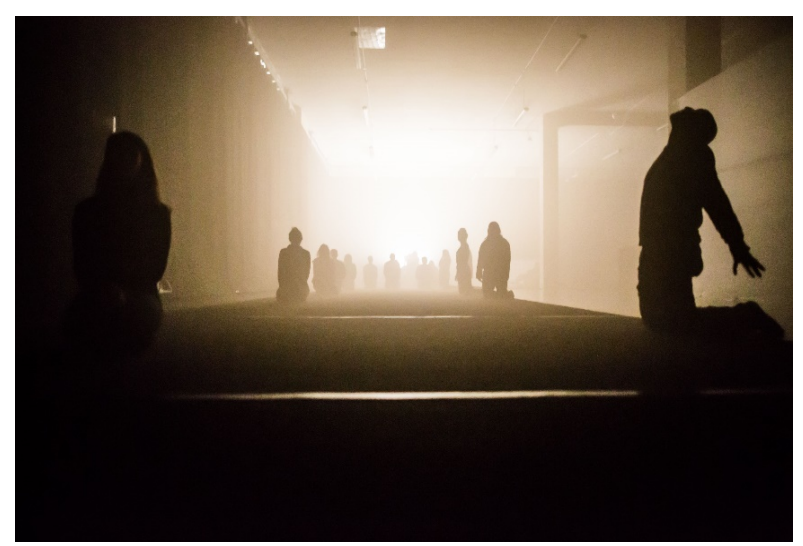

Figure 5: A guest in the third stage being fed to be controlled

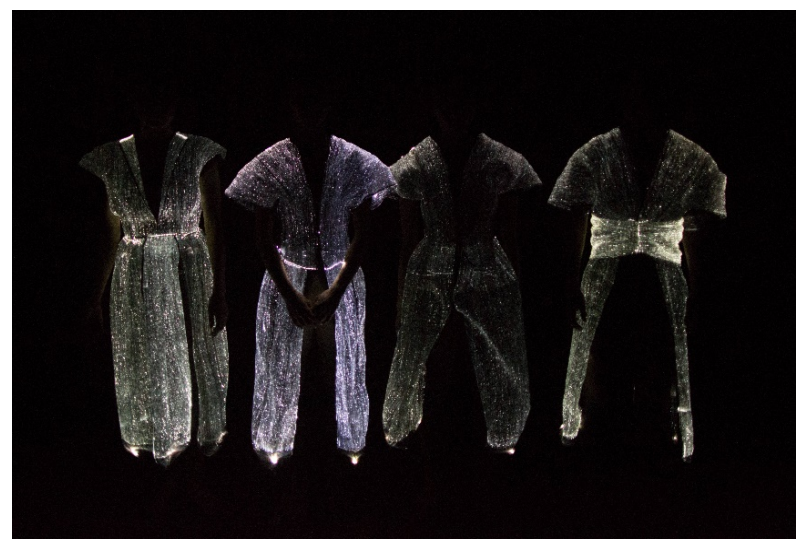

Figure 6: The four dancers in their light emitting "energy" dresses 